group (CG, $\mathrm{n}=52$ ). Tirofiban was only administrated in the tirofiban group. Before the CAG, enough clopidogrel, aspirin and heparin be used in both groups. The MACE and the haemorrhage events were collected in each group during in-hospital. The lesion and reperfusion of the IRA and myocardial were analyses by QCA and TMPG. The platelet aggregation rate were recorded All patients received UCG 1 week and 24 weeks after PCI to evaluate the heart function. Results There was no significant differences in age, gender, risk factors, pre-angina, the location of the AMI, heart function, and the mean interval from onset to PCI between the two groups. A greater percentage of TIMI 1 flow of IRA was achieved in TG compared with the control group before PCI $(p<0.05)$. The percentage of TIMI 3 flow of IRA after the guild wire first crossing was higher $(p<0.05)$ in TG. The percentage of TIMI 3 flow in TG after PCI was higher than that in CG $(p<0.05)$. The CTFC and slow-reflow phenomenon was fewer $(p<0.05)$ in TG after PCI. The percentage of TMPG beyond 2 grade was higher in TG $p<0.05)$. The value of LVEF 1 week after PCI in TG was higher than that in CG $(p<0.01)$. The platelet aggregation rate in TG was lower after tirofiban administration for $0.25,0.5,2,6$ and $12 \mathrm{~h}$. There was no significant difference in haemorrhage events between the two groups. There was a lower incidence of MACE in TG compared with that in CG during in-hospital and follow up.

Conclusion Intravenous administration of trofiban can inhibit the platelet aggregation, improve the coronary flow of IRA, decrease the incidence of NRP in AMI patients performed PCI, which in turn will improve the heart function and decrease the incidence of MACE. Tirofiban can make more IRA patent before PCI, but do not increase the haemorrhage events.

\section{e0656 THE EFFECT OF RECOMBINANT HUMAN B-TYPE NATRIURETIC PEPTID ON CORONARY CIRCULATION AND RENAL HAEMODYNAMICS IN YORK PIGS MODEL OF ACUTE MYOCARDIAL INFARCTION WITH HEART FAILURE}

doi:10.1136/hrt.2010.208967.656

Fu Xianghua, Zhang Jing, Wang Xuechao, Wang Yanbo, Xue Ling, Fan Weize, Wu Weili, Jiang Yunfa. The 2nd Hospital of Hebei Medical University, Shijiazhuang, Hebei, China

Objective To evaluate the impact of intravenous administration of rhBNP on coronary and renal artery haemodynamics in York pigs model of AMI-ADHF.

Methods 14 York pigs were included in this study. After the AMI$\mathrm{ADHF}$ models were established, pigs were randmized into saline group and rhBNP group. Coronary pressure $\left(\mathrm{P}_{\mathrm{c}}\right)$, the average peak velocity (APV), coronary vascular resistance (CR), coronary flow reserve (CFR) and coronary diameter were recorded simultaneously at baseline, instant after the model established, 60 min after continuous infusion of $0.01 \mu \mathrm{g} \cdot \mathrm{kg}^{-1} \cdot \mathrm{min}^{-1} \mathrm{rhBNP}$ and the time point of LVEDP $<12 \mathrm{~mm} \mathrm{Hg}$. The blood flow of the coronary were measured at rest and maximal hyperaemia. Renal angiography was performed by $4 \mathrm{~F}$ catheter and quantitative measurement of diameter was recorded by the computer assisting system. The average peak rate of renal artery $\left(\mathrm{APV}_{\mathrm{ra}}\right)$ was recorded, determination of quantitative angiography of renal artery diameter, renal vascular resistance. LVEDP and LVEF was measured.

Results 1. Coronary artery diameter increased after rhBNP administration. APV and CBF were significantly increased and CR decreased after rhBNP administration. CFR was significant rebound after continuous infusion of $0.01 \mu \mathrm{g} \cdot \mathrm{kg}^{-1} \cdot \mathrm{min}^{-1} \mathrm{rhBNP}$ for $30 \mathrm{~min}$. APV and CBF significantly increased and CR significantly decreased at the stage of infusion $0.010 \mu \mathrm{g} \cdot \mathrm{kg}^{-1} \cdot \mathrm{min}^{-1} \mathrm{rhBNP}$ in $\mathrm{rhBNP}$ Group. 2. Renal artery pressure was significantly lower after rhBNP administration. RhBNP exerts renal vasodilator effects in a dose related relationship. RBF increased gradually after administration of rhBNP and was significantly higher than control group. RVR decreased after administration of rhBNP. LVEF was lower than baseline after the models established and tended to increase after administration of rhBNP.

Conclusion It could increase blood flow of injury coronary artery, improve CFR and improve the coronary and renal haemodynamics after intravenous administration of rhBNP in pigs with AMI-ADHF.

\section{e0657 THE PERIOPERATION EFFECTS OF RECOMBINANT HUMAN B-TYPE NATRIURETIC PEPTIDE FOR HEART FAILURE PATIENTS WITH PRIMARY PERCUTANEOUS CORONARY INTERVENTION}

doi:10.1136/hrt.2010.208967.657

Fu Xianghua, Zhang Jing, Wang Xuechao, Wang Yanbo, Hao Guozhen, Fan Weize, Jiang Yunfa. The 2nd Hospital of Hebei Medical University, Shijiazhuang, Hebei, China

Objective To study the efficacy and safety of recombinant human Btype natriuretic peptide (rhBNP) in AMI-ADHF patients undergoing $\mathrm{PCI}$, especially changes in renal function and the impact of shortterm outcome during BNP treatment.

Methods 87 consecutive patients with AMI-ADHF entrolled in the study. All patients were randomly assigned to the rhBNP group and control group. rhBNP was given at $1.5 \mu \mathrm{g} \cdot \mathrm{kg}^{-1}$ intravenously and then infused intravenously $\left(0.0075-0.030 \mu \mathrm{g} \cdot \mathrm{kg}^{-1} \cdot \mathrm{min}^{-1}\right) .0 .9 \%$ Saline was used intravenously in control group as control. Clinical symptoms and killip grade were recorded. Plasma BNP levels were meaured before and after stopping the drug $6 \mathrm{~h}, 14$ days, 30 days. LVEDD and LVEF was measured. Serum creatinine (Scr) was measured before and after administered the medication $24 \mathrm{~h}, 48 \mathrm{~h}$, $72 \mathrm{~h}, 7$ days and 14 days using simplified MDRD equation to calculate estimated glomerular filtration rate. Recording the major adverse cardiac events occurrence within 30 days.

Results rhBNP group has a less dyspnoea time than the control group; The plasma BNP levels significantly lower than before treatment at different time point in the two groups. The LVEF was significantly higher in treatment group compared with baseline levels after treatment $24 \mathrm{~h}$, while LVEDD significantly decreased even after discontinuation the treatments, which remain so when the 30 days. The LVEF and LVEDD improvements in rhBNP group were significantly better than in the control group after treatment 24 h, 14 days. At day 7 after PCI, the SCr had lowered to the baseline level in the rhBNP group. The estimated glomerular filtration rate after PCI was higher in the rhBNP group than that in the control group. The occurrence of CIN was significantly lower in the rhBNP group than in the control group. The MACE event of $30 \mathrm{~d}$ in rhBNP group was significantly lower than the control group.

Conclusion rhBNP can promptly and effectively improve the heart function, reduce the incidence of major adverse cardiac events rate in acute myocardial infarction with heart failure patients, which also had a renal function protective effect in patients with and decreased incidence on CIN.

\section{e0658 ESTABLISHMENT OF YORK PIG MODEL OF ACUTE MYOCARDIAL INFARCTION WITH ACUTE DECOMPENSATED HEART FAILURE BY CORONARY OCCLUSION WITH BALLOON AND INJECTING OF MICROEMBOLUS}

doi:10.1136/hrt.2010.208967.658

Fu Xianghua, Zhang Jing, Wang Xuechao, Wang Yanbo, Miao Oing, Jiang Yunfa, Hao Guozhen, Gu Xinshun. The 2nd Hospital of Hebei Medical University, Shijiazhuang, Hebei, China

Objective To evaluate the method of yorkpig model of AMI-ADHF by coronary occlusion with balloon and injecting of microembolus 